\title{
O que sabemos dos grupos construtores de sambaquis? Breve revisão da arqueologia da costa sudeste do Brasil, dos primeiros sambaquis até a chegada da cerâmica Jê
}

\author{
Ximena S. Villagran *
}

\begin{abstract}
VILLAGRAN, X.S. O que sabemos dos grupos construtores de sambaquis? Breve revisão da arqueologia da costa sudeste do Brasil, dos primeiros sambaquis até a chegada da cerâmica Jê. R. Museu Arq. Etn., São Paulo, n. 23, p. 139-154, 2013.
\end{abstract}

Resumo: Desde a década de 1990 até o ano de 2012, mais de cento e cinquenta trabalhos foram produzidos sobre a pré-história do litoral sudeste do Brasil. A grande maioria dos trabalhos refere-se aos sítios sambaquis, outra porção aos denominados "acampamentos litorâneos", com e sem cerâmica, que caracterizam a pré-história mais recente do litoral. Dentro de tão vasto volume de trabalhos, quanto sabemos atualmente sobre a evolução da ocupação humana da costa e as características dos grupos que lá habitaram antes da migração massiva dos Guaranis? Este trabalho pretende sintetizar as informações mais relevantes produzidas nos últimos vinte anos sobre a pré-história do litoral sudeste e aventar novas hipóteses para discutir velhas questões referentes ao povoamento da costa e à "desaparição" dos grupos construtores sambaquis.

Palavras-chaves: Arqueologia costeira; Povoamento da costa; Acampamentos litorâneos; Cerâmica Taquara/Itararé.

\section{Introdução}

$\mathrm{N}_{\mathrm{p}}^{\infty}$

os últimos vinte anos numerosas pesquisas abordaram a arqueologia da costa sudeste do Brasil, com especial ênfase nos sítios sambaquis. Os trabalhos referem-se a resultados de análises em sítios específicos,

(*) Universidade de São Paulo - Instituto de Geociências <villagran@usp.br>

Eberhard Karls Universität Tübingen - Institut für Natturwissenchaftliche Archäeologie principalmente estudos de dieta e novas abordagens metodológicas, assim como datações que recuam na antiguidade da ocupação litorânea até o inicio do Holoceno. Desde a década de 1990 até o ano de 2012, cerca de cento e cinquenta trabalhos foram produzidos sobre arqueologia, bioantropologia e geoarqueologia de sambaquis e sítios costeiros com cerâmica Jê. Esse número inclui artigos científicos, teses e dissertações defendidas no Brasil e no exterior, assim como artigos de revisão sobre projetos de pesquisa. Porém, o número pode subestimar a verdadeira produção científica sobre o tema, já que potencialmente existem trabalhos de formatura e ar- 
O que sabemos dos grupos construtores de sambaquis? Breve revisão da arqueologia da costa sudeste do Brasil, dos primeiros sambaquis até a chegada da cerâmica Jê

R. Museu Arq. Etn., São Paulo, n. 23, p. 139-154, 2013.

tigos publicados em revistas locais que também contribuem à enorme produção relacionada com a pré-história da costa sudeste.

O fato de contar com tão vasta quantidade de trabalhos e pesquisas aporta, por um lado, à produção de conhecimento sobre as culturas litorâneas, mas pode, por outro, gerar uma nuvem de informações dispersas de difícil articulação na construção do passado. Portanto, cabe perguntar, com tanta produção associada a uma única temática, quanto se sabe, de fato, sobre as culturas litorâneas do Brasil, sua antiguidade e sua evolução ao longo do Holoceno? Quanto se sabe sobre o grupo humano por trás dos volumosos e conspícuos sambaquis que capturaram a atenção de amadores e cientistas desde o século XIX? Quanto se sabe sobre a desaparição destes grupos do registro arqueológico?

A proposta deste trabalho é revisar e sintetizar os conhecimentos produzidos por dezenas de pesquisadores nos últimos vinte anos sobre as características da ocupação humana da costa sudeste do Brasil. A tentativa é de sintetizar as informações sobre cronologia, padrão de assentamento, dieta e cotidiano e avançar na discussão sobre o povoamento do litoral e a desaparição dos sítios associado às tradicionais culturas da costa. O contexto cronológico vai dos sambaquis mais antigos até o momento em que se detecta a presença de cerâmica Jê no litoral sudeste.

Não se pretende com esta revisão esgotar as possibilidades interpretativas que derivam do volumoso conjunto de pesquisas realizadas nos últimos vinte anos sobre o tema. Este compêndio aspira a agrupar os dados e referências bibliográficas a modo de ordenar as informações e estabelecer vínculos entre novas análises, novos resultados e velhas discussões.

\section{Os primeiros sambaquis e a ocupação da costa}

Os registros sobre ocupação sambaquieira são mais numerosos nos estados de Santa Catarina (região de Laguna, Florianópolis e Joinville), Paraná (baías de Paranaguá e Guaratuba), São Paulo (Cananeia-Iguape e Santos) e Rio de
Janeiro (baía de Guanabara e Região dos Lagos). No entanto, numerosos sambaquis também se encontram nos estados de Paraná e Espírito Santo, por exemplo, onde as pesquisas foram menos abundantes.

Atualmente, propõe-se que teriam existido dois blocos maiores, embora não exclusivos, de desenvolvimento da cultura sambaquieira: 1) Rio de Janeiro, onde os sítios são de mediano porte (até $5 \mathrm{~m}$ de altura), com associação entre áreas de habitação e locais de sepultamento (Gaspar, 1991; Barbosa, 2001, 2007); 2) Santa Catarina, onde os sítios são frequentemente monumentais (mais de $5 \mathrm{~m}$ de altura), com maior quantidade de sepultamentos humanos e presença de zoólitos (Fish et al., 2000; Gaspar et al., 2008; Gaspar, 1998).

Alguns autores propõem que estas duas áreas corresponderiam a dois prováveis eixos, norte e sul, respectivamente, de povoamento do litoral brasileiro desde o interior. O local de origem desta leva migratória estaria localizado entre o norte do Estado de Paraná e sul de São Paulo, segundo Neves (1988), ou entre o norte do Estado de Santa Catarina e o sul do Paraná, segundo Oliveira (2000). Porém, os sambaquis antigos, do início do Holoceno, não se encontram no litoral paranaense, onde a data mais recuada alcança os 6500 anos AP (Garcia 1979 em Brochier, 2009), mas sim nos estados de São Paulo, Rio de Janeiro e Santa Catarina. No entanto, deve-se lembrar de que apesar de terem sido prospectados mais de 300 sambaquis no litoral do Paraná, somente 13 possuem datação (Brochier 2009).

Os sambaquis mais antigos correspondem, respectivamente, aos sítios Cambriu Grande, datado em ca. 7.800 anos cal. AP (Calippo, 2004), e o sambaqui do Algodão, datado em ca. 8.700 anos cal. AP (Lima et al., 2002). No estado de Santa Catarina, o sambaqui mais antigo, o sítio Caipora, está localizado a aproximadamente 20 $\mathrm{km}$ da atual linha de costa e foi datado em ca. 7400 anos cal. AP (Giannini et al., 2010).

Sambaquis mais antigos ainda se encontram em áreas interioranas associadas a contextos fluviais no estado de São Paulo. O sítio Capelinha, de pouco mais de 10.000 anos cal. AP é o sambaqui fluvial mais antigo do vale 
do rio Ribeira de Iguape (Neves et al., 2005). A existência de sambaquis fluviais datados do início do Holoceno levou à tentadora possibilidade de acreditar que os grupos litorâneos derivam geográfica e culturalmente de pioneiros coletores de moluscos vindos do interior. No entanto, nos últimos vinte anos o estudo da relação entre sambaquis costeiros e fluviais, desde diversas abordagens, coloca as duas realidades como potencialmente derivadas do mesmo grupo e/ou cultura. Esse grupo teria habitado, no final do Plesitoceno, a plataforma continental hoje submersa.

\section{A relação com os sambaquis fluviais}

As evidências arqueológicas e bioantropológicas indicam que os grupos sambaquieiros do litoral atlântico formam uma entidade independente, que não guardaria relação evolutiva, genética nem cultural, com os grupos que atualmente ocupam o planalto. Os sambaquieiros, sim, estão vinculados com as populações ribeirinhas que exploraram igualmente moluscos terrestres, no começo do Holoceno. Os grupos associados à formação dos sambaquis fluviais do vale do Ribeira de Iguape, apesar de subsistirem de espécies faunísticas e vegetais da Mata Atlântica e não litorâneas (ver Alves, 2008 e Plens, 2007), apresentam afinidade biológica (Neves and Okumura, 2005; Bartolomucci, 2006; Okumura, 2007) e cultural com os grupos costeiros. Outros trabalhos bioantropológicos mostram diferenças entre os indivíduos sepultados nos sambaquis fluviais de São Paulo e aqueles enterrados em sambaquis costeiros desse estado e de Santa Catarina (Filippini, 2004; Filippini and Eggers, 2005). No entanto, nestes trabalhos não se discutem as causas da diferenciação e inclusive se menciona a possibilidade das diferenças serem produto do isolamento geográfico de populações de origem semelhante.

A relação entre sambaquis litorâneos e fluviais é frequentemente explicada como resultado de intrusões sazonais dos grupos litorâneos até o interior. Lima (1999) coloca a ocupação do Vale do Ribeira como resultado de intrusões oportunistas de grupos eficientemente adaptados à vida no litoral. Essas intrusões teriam seguido os vales fluviais que cortam a serra do Mar e atuam como portais naturais que facilitam o deslocamento entre a costa e o interior (Lima, 1999; Lima \& Lopez Mazz, 1999). Uma hipótese semelhante foi proposta por Calippo (2010), que também defende a relação entre sambaquis fluviais e litorâneos do Estado de São Paulo, com uma possível origem comum vinculada à ocupação dos paleovales fluviais e paleoplanícies litorâneas do Pleistoceno, como também proposto por Barreto (1988). Essa relação original teria desembocado, ao longo do tempo, em uma diferenciação entre os grupos que permaneceram no ambiente fluvial e os que se especializaram no ambiente litorâneo.

\section{A rota litorânea}

Os sítios concheiros, a exemplo dos sambaquis brasileiros, estão entre as manifestações mais antigas de ocupações litorâneas com economias marítimas nas Américas. No litoral pacífico do Canadá, Estados Unidos e Peru existem concheiros a céu aberto e em caverna com idades máximas de até 12.000 anos AP (Cannon, 2000; Sandweiss, 2003; Erlandson et al., 2007). No entanto, as evidências de economias marítimas antecedem estas ocupações em até dois mil anos. Tal é o caso do sítio Monte Verde, na costa sul do Chile, datado em ca. 14.500 anos cal. AP (Dillehay, 1999) e do sítio Huaca Prieta, na costa norte do Peru, datado em ca. 14.200 anos cal. AP (Dillehay et al., 2012).

As evidências de ocupações costeiras anteriores ao início do Holoceno são usadas para reforçar a hipótese de uma via litorânea de chegada de grupos humanos às Américas durante o Pleistoceno (entre 20 e 15 kA) através da borda do Pacífico (Fladmark, 1979; Dixon, 2001; Erlandson, 2001; Mandryk et al., 2001; Erlandson et al., 2007; Fagundes et al., 2008). Dados geológicos e biológicos mostram que a costa oeste da América do Norte era propícia para sustentar o deslocamento de grupos humanos desde, pelo menos, 16.000 anos cal. AP (Dixon, 2013). Alguns autores propõem que, uma vez na América do Sul, a migração costeira teria seguido uma rota atlântica e outra pacífica de colonização, com ingresso ao interior do con- 
O que sabemos dos grupos construtores de sambaquis? Breve revisão da arqueologia da costa sudeste do Brasil, dos primeiros sambaquis até a chegada da cerâmica Jê

R. Museu Arq. Etn., São Paulo, n. 23, p. 139-154, 2013.

tinente pelos vales fluviais que desembocavam no oceano (Dixon, 2001; Hubbe et al., 2011; Miotti \& Salemme, 2004; Miotti, 2003; Neves et al., 2003).

Da suposta rota atlântica, as ocupações litorâneas mais antigas do Brasil podem ser remanentes. Nesse sentido, as pesquisas sobre sambaquis na plataforma continental submersa (ver Calippo 2010), ou sobre sítios soterrados por depósitos holocênicos (ver Brochier 2009), podem ainda trazer ocorrências mais antigas que corroborem, ou refutem, os modelos de dispersão existentes.

Vários indícios já apontam a possível existência de uma antiga via de chegada e ocupação da costa brasileira, de origem exclusivamente litorânea, como, por exemplo: 1) a falta de relações genéticas entre indivíduos do interior e litoral (ver Okumura 2007); 2) as diferenças na cultura material dos grupos do interior e dos grupos litorâneos que, por sua vez, apresentam cultura lítica de características semelhantes ao longo de toda a franja costeira (DeBlasis et al., 1998; Tenório, 2004); 3) e o vinculo genético e cultural entre os grupos litorâneos e aqueles que ocuparam um dos corredores mais importantes de comunicação entre o planalto e litoral (os sambaquis fluviais do vale do Ribeira de Iguape) (ver Calippo 2010; Lima, 1999; Lima \& Lopez Mazz, 1999; Okumura 2007). Somado a isso, a lacuna de datas recuadas para o estado do Paraná (suposta via de entrada do interior à costa), apesar de ser potencialmente uma ausência metodológica, como mencionado antes, indiretamente sustenta uma via litorânea de migração.

O povoamento de América do Sul ainda é objeto de controvérsia. Ainda não há provas suficientes que afirmem movimentos desde o interior do continente até a costa ou vice-versa; assim como não há consenso quanto à quantidade de fluxos migratórios que existiram da Ásia até as Américas (Dillehay, 2009). Assim, a maneira de hipótese pode-se pensar que a ocupação da costa brasileira possa estar relacionada com uma potencial rota atlântica de colonização da América do Sul (ver Hubbe et al., 2011; Neves et al., 2003; Rothhammer \& Dillehay, 2009). Os seus remanentes estariam hoje submersos devido ao aumento do NRM durante o Pleistoceno e Holoceno. A relação entre a dinâmica costeira e a potencial localização de sambaquis antigos é explicada graficamente por Calippo (2010), que propõe um modelo de paleolinhas de costa com áreas possíveis de localização de sítios costeiros datados de finais do Pleistoceno.

\section{Os sambaquieiros como sistema cultural}

A associação entre área de moradia, descarte de alimentos e sepultamentos humanos em sambaquis, definidos como espaços multifuncionais (Gaspar, 1991), levou a considerar aos sambaquieiros como único sistema ou unidade cultural de grande expansão ao longo da costa brasileira, embora com desenvolvimentos regionais específicos (Gaspar, 1991, 1998, 2000; Tenório, 2004). Inclusive a cultura material dos grupos sambaquieiros permanece constante ao longo da faixa costeira, caracterizada, em termos gerais, por tecnologia lítica e óssea polida (DeBlasis et al., 1998; Tenório, 2004).

No entanto, as diferenças morfológicas, composicionais e inclusive artefatuais, observadas por trás da aparente homogeneidade dos sambaquis, também foram propostas como evidência de uma multiplicidade de sistemas socioculturais que se expandiram ao longo da costa (Lima, 1991, 1999). Nesse sentido, estudos bioantropológicos mostram a homogeneidade genética entre os indivíduos sepultados em sambaquis, com existência de microdiferenciações regionais na costa (Neves, 1988; Mendonça de Souza, 1995; Okumura, 2007). Também estudos isotópicos, realizados em sambaquieiros do litoral paulista, identificaram microdiferenciações, explicadas como produto do desenvolvimento local de grupos que habitaram a plataforma continental hoje submersa (Calippo, 2010). Isso sugere que tanto unidade quanto desenvolvimentos locais podem expressar a realidade biológica e cultural da ocupação costeira associada à formação dos sambaquis.

As pesquisas de tipo sistêmico-funcionais realizadas em sambaquis (ver Villagran, 2010) produziram informações de base que permitiram caracterizar os grupos costeiros quanto ao 
seu padrão de assentamento, função dos sítios, subsistência e organização social. $\mathrm{O}$ assentamento teria sido preferencial em franjas do litoral que incluíram a formação de estuários, baías e sistemas de lagunas costeiras durante sua evolução no Holoceno (Gaspar, 1991; Figuti, 1993; DeBlasis et al., 1998; Lima, 1999; Lima and Lopez Mazz, 1999; Fish et al., 2000; Amâncio and Dominguez, 2003; Perreti, 2009; Calippo, 2010). A distribuição dos sítios formaria grupamentos ao redor dos grandes corpos de água, fonte dos recursos alimentícios (Gaspar, 1991; Kneip, 2004; Barbosa, 2007). A ocupação dos sambaquis teria sido contínua, sem táticas sazonais de mobilidade, com sítios que serviam de local de residência contínua e outros que eram ocupados como acampamento temporário (De Masi, 1999, 2001).

Apesar do volume de moluscos acumulados nos sambaquis, os peixes seriam a principal fonte de proteína para estas populações (Bandeira, 1992; Figuti \& Klokler, 1996; Figuti, 1992, 1993; Klokler, 2001, 2008; De Masi, 1999, 2001), com consumo complementar de espécies vegetais (Boyadjian 2007; Scheel-Ybert et al. 2003, 2009a; Wesolowski 2000; Wesolowski et al. 2007) e consumo ocasional de mamíferos marinhos (focas, golfinhos e baleias) (Castilho, 2005; Castilho \& Simões-Lopes, 2008; Klokler, 2008; Prous, 1992). Os conjuntos artefatuais recuperados em sambaquis, como arpões e redes de pesca, confirmam a dependência dos recursos aquáticos (Prous, 1992; Tiburtius, 1996; DeBlasis et al., 1998).

A existência de embarcações para deslocamento e pesca foi proposta por Gaspar (1991) e inferida de maneira indireta em vários trabalhos posteriores. As inferências baseiam-se na existência de sambaquis em ilhas, paleoilhas hoje ancorados à terra firme por tômbolos, ou em morros testemunho do embasamento cristalino que antigamente se achavam em meio a grandes corpos de água atualmente assoreados (Amenomori, 2005; Calippo, 2010; Giannini et al., 2010; Nishida, 2001; Prous, 1992; Tenorio, 2003).

Outra vertente de estudos, que inclui aos trabalhos especializados sobre componentes particulares dos sítios, permitiu refinar aspectos relacionados com as atividades cotidianas dos grupos costeiros. Estudos bioantropológicos confirmaram a prática de pesca e mergulho através de marcadores osteológicos específicos, tanto em homens quanto em mulheres (Neves, 1988; Mendonça de Souza, 1995; Rodrigues-Carvalho, 2003; Okumura and Eggers, 2005; Petronilho, 2005), junto com evidências de sedentarismo e alta densidade populacional (Mendonça de Souza, 1995; Neves and Wesolowski, 2002; Okumura and Eggers, 2005).

Quanto aos padrões de residência, Hubbe et al. (2009) corroboraram a proposta de Neves (1998) da existência de um padrão de residência matrilocal entre os sambaquieiros. Os autores baseiam sua conclusão em uma amostragem extensa que inclui tanto sambaquis, como sítios com cerâmica Taquara/Itararé nos estados de Paraná, Santa Catarina e Rio Grande do Sul.

Por último, os trabalhos de influência pós-moderna na arqueologia de sambaquis, que apontam a aspectos simbólicos do ritual funerário associado à formação de alguns sítios, mostram a relação dos ambientes costeiros e seus recursos no ritual funerário e simbolismo dos grupos litorâneos (Gaspar, 2000, 2004; Gaspar et al., 2007; Klokler, 2008). Dentre estes trabalhos, destaca-se uma nova linha de pesquisa que se refere a estudos de gênero. $O$ estudo pioneiro foi realizado por Escórcio \& Gaspar (2010) a partir da análise do mobiliário funerário em homens e mulheres sepultados em sambaquis do litoral do Rio de Janeiro. As autoras indicam a ausência de divisões rígidas entre os gêneros e grande variabilidade nas frequências de tipos artefatuais associados a cada gênero. Nesse sentido, cabe ressaltar as altas ocorrências de lesões ósseas observadas em mulheres enterradas no sambaqui Zé Espinho, litoral do Rio de Janeiro, interpretada como indicador de violência doméstica ou sequestro (Rodrigues-Carvalho et al., 2009).

Essas informações permitem definir os grupos sambaquieiros como culturas marítimas, como estabelecido por Calippo (2010) a partir das propostas de Muckelroy (1978), Adams (2002) e Diegues (2004). A relação dos sambaquieiros com as fontes de recursos aquáticos (mar, lagunas, estuários) iria além da exploração 
O que sabemos dos grupos construtores de sambaquis? Breve revisão da arqueologia da costa sudeste do Brasil, dos primeiros sambaquis até a chegada da cerâmica Jê

R. Museu Arq. Etn., São Paulo, n. 23, p. 139-154, 2013.

dos seus componentes bióticos para subsistência do grupo. A exploração dos ambientes litorâneos comportaria uma visão específica do mundo, desenvolvida a partir do vínculo e da "apropriação" não exclusivamente econômica, mas também política, social e simbólica do meio físico e suas particularidades.

\section{A formação dos sambaquis}

Desde o abandono do debate inicial sobre o caráter antrópico ou natural dos sambaquis, a intencionalidade na formação dos sítios tornou-se essencial nas explicações sobre a formação dos sítios. Na década de 1990, vários autores postularam que os sambaquis podem ser considerados como artefatos, por terem sido deliberadamente construídos a partir da acumulação humana de conchas (Gaspar, 1991; Gaspar and DeBlasis, 1992; Afonso and DeBlasis, 1994; Figuti and Klokler, 1996).

Atualmente considera-se que as conchas que constituem a matriz dos sambaquis teriam sido utilizadas, na sua maioria, como elemento construtivo que outorga volume às estruturas, utilizadas tanto como local de habitação quanto como de deposição dos mortos (Afonso \& DeBlasis, 1994; Figuti \& Klokler, 1996; Gaspar, 1991, 2004; Gaspar et al., 1994; Gaspar \& DeBlasis, 1992; Klokler, 2001, 2008). Inclusive a escolha dos locais de coleta das conchas nem sempre estaria associada à proximidade do recurso, senão a preferências culturalmente determinadas (Calippo, 2010).

Diversas hipóteses e modelos foram propostos para explicar o processo de construção dos sambaquis. Em todos os modelos predomina a visão dicotômica centro versus periferia, como estrutura subjacente à ordenação do espaço nos sítios. Áreas centrais de habitação, caracterizadas por grandes estruturas de combustão ou pisos de argila, estariam rodeadas ou seriam imediatas ao local de deposição e acumulação de resíduos cotidianos, fato caracterizado também pela maior frequência de resíduos de lascamento (Gaspar and DeBlasis, 1992; Bryan, 1993; Tenório et al., 2008).

O sambaqui com informações mais detalhadas sobre o seu processo de formação e arquitetura é o sítio Jabuticabeira 2, localizado no litoral sul de Santa Catarina. Composto por duas camadas estratificadas de espessura métrica (conchífera na base e ictiológica no topo), este sambaqui ofereceu as seguintes informações e interpretações: 1) a construção do sítio seguiu um padrão de levantamento de pequenos montículos funerários, que se superpõem no tempo e no espaço (DeBlasis et al., 1998; Fish et al., 2000; Karl, 2000; Klokler, 2001, 2008; Bendazzoli, 2007; Nishida, 2007); 2) os sepultamentos humanos eram acompanhados por resíduos de festins celebrados em honra aos mortos (Klokler 2001, 2008); 3) a queima de materiais vegetais e faunísticos, de espécies vegetais e animais disponíveis nas proximidades do sítio, foi uma prática recorrente (Bianchini, 2008; Klokler, 2008; Nishida, 2007; Villagran, 2008; Villagran et al., 2009, 2010) e algumas espécies vegetais podem ter sido queimadas graças a suas características aromáticas, vinculadas com atividades rituais no sambaqui (Bianchini et al., 2007); 4) os materiais que compõem tanto a camada conchífera como a ictiológica representam uma somatória de episódios de queima, acumulação e descarte de resíduos alimentares para a construção dos montículos, através de um processo que expressa a ritualização dos elementos descartados em contexto doméstico ou cerimonial (Villagran, 2008, 2010; Villagran et al., 2009; Villagran et al., 2009a; Villagran et al., 2010). Nesse sentido, a ausência de áreas de habitação claramente identificáveis no sambaqui ou nas imediações pode estar vinculada ao hábito retrabalhamento de resíduos para posterior deposição no sambaqui (Villagran 2008, 2010; Villagran et al. 2009a, 2009b).

Em termos gerais pode-se dizer que os modelos propostos para a formação dos sítios derivam do tipo de intervenção e análise realizada em cada caso. Sítios onde houve escavações amplas costumam fornecer informações mais detalhadas sobre a sua função; enquanto sítios como Jabuticabeira 2, onde as análises se baseiam em amostragens nos perfis estratigráficos, permitem observar a espacialidade e temporalidade dos padrões construtivos, assim como a continuidade ou mudança de comportamento na atividade construtora. 


\section{Os “acampamentos conchíferos/ litorâneos"}

As evidências de ocupação humana pré-histórica no litoral sul e sudeste do Brasil não se restringem aos sambaquis. Existe outro tipo de sítio na costa, denominado de "acampamento litorâneo" e formado por acumulações rasas (de menos de $1 \mathrm{~m}$ de altura) e superfície variável (de 200 até $2000 \mathrm{~m} 2$ ) de sedimentos finos, frequentemente referidos como "terra preta", ossos de peixe e escasso, senão nulo, conteúdo de conchas de moluscos.

Os acampamentos conchíferos ou litorâneos, também referidos na literatura mais antiga como paradero, sítio paleoetnográfico, sítios rasos, ou sambaquis sujos, podem acontecer seja como sítio isolado, seja superposto a sambaquis (Lima 1999; Prous 1992). Esse tipo de depósito encontra-se desde o Rio de Janeiro até o Rio Grande do Sul. No Rio de Janeiro foram associados à tradição Itaipu (Dias, 1976; Mendonça de Souza, 1991), esquema classificatório atualmente em desuso (Gaspar 1991).

Em São Paulo encontram-se nas ilhas e apresentam associação entre área de moradia e sepultamento dos mortos (Nishida, 2001; Amenomori, 2005). No Paraná, as informações são escassas e incluem uma menção no trabalho de Bigarella (1950). Em Santa Catarina os acampamentos litorâneos são abundantes, especialmente na Ilha de Santa Catarina e no litoral norte do estado. Apresentam vastas áreas habitacionais, com estruturas de combustão (rodeadas de seixos), buracos de estaca, pisos de argila compactada, "fornos polinésios” e artefatual lítico e ósseo. Grandes concentrações de sepultamentos humanos são igualmente comuns (Beck, 1971, 1972; Rohr, 1984; Prous, 1992).

Por serem compostos primordialmente de restos de peixe, os acampamentos litorâneos foram tradicionalmente interpretados como produto de uma mudança na dieta das populações costeiras. Essa mudança teria envolvido a substituição da coleta de moluscos pelo desenvolvimento de pesca especializada, motivada por câmbios ambientais e/ou superexploração dos bancos de moluscos (Lima, 1991, 1999; Schmitz et al., 1993; Mendonça de Souza, 1995). Na mesma linha interpretativa, a nova estratégia de subsistência indicaria a transformação dos coletores de moluscos em grupos mais sedentários, com economia de pesca e organização de tipo tribal (Lima, 1999).

No entanto, estudos zooarqueológicos (Bandeira, 1992; Figuti, 1992; Nishida, 2001, 2007) e isotópicos (De Masi 1999, 2001) demonstraram que a pesca foi a atividade econômica principal tanto em sambaquis como em acampamentos litorâneos, e o consumo de peixe a fonte básica de proteína entre todos os grupos costeiros.

A diferença na distribuição geográfica entre sambaquis, associados aos sistemas lagunares, e os acampamentos, frequentemente localizados em praias de mar aberto, pode resultar de um viés de observação e/ou preservação; isto é, a baixa visibilidade dos sítios superficiais e sua suscetibilidade ao preenchimento sedimentar característico das porções do litoral brasileiro submetidas ao efeito das variações do NRM no Holoceno (Brochier, 2009; Giannini et al., 2010). Isso se vincula com a cronologia dos sítios, já que a idade máxima dos acampamentos, em torno aos 2600 anos AP (Schmitz et al., 1992; Schmitz and Bittencourt, 1996; Nishida, 2001; Barbosa, 2007), é sempre posterior à máxima transgressão holocênica.

Estudos craniométricos mostraram afinidade genética entre os indivíduos sepultados nos acampamentos das ilhas paulistas e nos sambaquis do litoral continental do estado, mesmo padrão observado entre acampamentos na ilha de Santa Catarina e sambaquis continentais (Okumura, 2007). No Estado de São Paulo, a afinidade biológica teria se transformado, com o tempo, em estratégias de subsistência diferenciadas para ocupações em sambaquis e acampamentos (ver Calippo 2010). A associação entre áreas de moradia, descarte de alimentos e enterramento dos mortos que se observa em sambaquis e acampamentos litorâneos, junto com a similaridade nas indústrias líticas, ósseas e malacológicas e afinidade biológica nos indivíduos sepultados em ambos os tipos de sítio, sugere que sambaquis e acampamentos teriam sido produzidos por uma mesma população e que se tratava de realidades coetâneas. 
O que sabemos dos grupos construtores de sambaquis? Breve revisão da arqueologia da costa sudeste do Brasil, dos primeiros sambaquis até a chegada da cerâmica Jê

R. Museu Arq. Etn., São Paulo, n. 23, p. 139-154, 2013.

\section{Por que desapareceram?}

Os processos que conduziram ao fim da cultura sambaquieira compreendem uma combinação de fatores ambientais e culturais. Entre os fatores ambientais propõem-se: o impacto que teve nos bancos de moluscos a mudança na salinidade dos corpos de águas devido às flutuações do nível relativo do mar no Holoceno (Barbosa, 2007; Calippo, 2010; Gaspar et al., 2008; Hurt, 1974); e a predação antrópica dos moluscos, que teria provocado a completa exaustão deste recurso (Lima 1999). Os fatores culturais referem-se à migração até o litoral dos grupos ceramistas do tronco linguístico Jê, vindos do planalto há aproximadamente 1500-1000 anos atrás, o que teria provocado a completa desestabilização do sistema sociocultural dos sambaquieiros e posto fim ao projeto de acumulação social de conchas (Beck, 1972; Gaspar, 1991; Schmitz et al., 1993; Lima, 1999; Barbosa, 2007; DeBlasis et al., 2007; Gaspar et al., 2007, 2008) de modo aparentemente violento e conflituoso (Lessa, 2005; Lessa \& Scherer, 2008).

A influência dos grupos Jê no litoral observa-se claramente na existência de sítios costeiros com cerâmica Taquara/Itararé, definidos aqui como "sítios mistos". Esses sítios podem aparecer isolados ou nos estratos superiores dos sambaquis (Schmitz et al., 1993; DeBlasis et al., 1998; Lima, 1999; Gaspar et al., 2008). A cerâmica Taquara/Itararé caracteriza os grupos Jê meridionais, que teriam chegado ao sul do Brasil, desde o planalto central brasileiro, há pelo menos 3000 anos (Brochado, 1984; Urban, 1992; Noelli, 2000). Os sítios mistos são frequentemente datados entre 1500 e $500 \mathrm{AP}$ e têm a particularidade de apresentar, juntamente com cerâmica Taquara/Itararé, ritual funerário e conteúdo faunístico e artefatual semelhantes aos da cultura sambaquieira. Exemplo disso são: a camada superficial dos sambaquis Enseada I e Forte Marechal Luz; e os sítios Praia das Laranjeiras II, Tapera, Base Aérea e Armação do Sul (Beck, 1972; Schmitz et al., 1992, 1993).

Esses sítios representam a intensificação do contato entre planalto e litoral, já que evidências de intercâmbio material entre as duas regiões, sem intercâmbio genético (ver Okumura, 2007), existem desde antes da aparição dos sítios mistos.
Por exemplo, indústria lítica típica do planalto foi achada em sítios costeiros, assim como zoólitos também foram achados em sítios do planalto (Silva et al., 1990; Prous, 1992). Grânulos de amido de araucária também foram achados no cálculo dentário de indivíduos sepultados em sambaquis (Wesolowski et al., 2007). No Rio de Janeiro, fragmentos de cerâmica Una (também associada ao tronco Macro-Jê) foram recuperados em sambaquis em níveis de até 3600 anos AP (Crancio and Kneip, 1994; Kneip, 1994).

No próximo tópico, se discutirão os estudos mais recentes realizados em sítios mistos do Estado de Santa Catarina e como as evidências assinalam a necessidade de formular novas explicações para a influência Jê no litoral.

\section{Os sítios "mistos" ou sítios costeiros com cerâmica}

Os sítios mistos aparecem em maior número no Estado de Santa Catarina, apesar de também existirem nos estados de Paraná e Rio Grande do Sul, embora em menor frequência de aparição. Esses sítios foram tradicionalmente associados à chegada dos grupos ceramistas que, uma vez na costa, teriam adotado estratégias de captação de recursos que incluíam a pesca e coleta de moluscos (Silva et al., 1990; Schmitz et al., 1993; Schmitz, 1996, 1999; Bandeira, 1999; Fossari, 2004). A interpretação baseia-se na diferença morfológica e composicional entre sítios mistos e sambaquis, junto com a presença de cerâmica e maior diversidade na indústria óssea que se observa em alguns sítios mistos. No entanto, estudos recentes sugerem um panorama algo distinto.

Wesolowski (2000), a partir de análise de marcadores osteológicos, observa padrão típico de grupos caçadores-coletores tanto em indivíduos sepultados em sambaquis como em sítios mistos do litoral norte de Santa Catarina. Existiria uma continuidade na dieta dos habitantes da costa, sem adoção da horticultura quando da chegada da tecnologia cerâmica. Isso leva a autora a construir duas hipóteses: 1) que os grupos que ocuparam os sítios mistos não guardariam relação com os grupos do planalto; 2) que os grupos do planalto, horticultores, abandonaram esta prática ao chegar ao litoral. 
Análises craniométricas realizadas por Okumura (2007) reafirmam o proposto por Neves (1988) sobre a afinidade genética entre indivíduos sepultados em sítios mistos e sambaquis da Ilha de Santa Catarina. Os mesmos estudos mostram semelhanças entre séries ceramistas e sambaquis (não ceramistas) do litoral norte e sul do estado. Já no litoral norte e sul de Santa Catarina, a continuidade genética não é tão clara (Okumura 2007).

As análises isotópicas realizadas por Bastos (2009) e Bastos et al. (2011) em indivíduos sepultados nos níveis pré-cerâmicos e cerâmicos do sambaqui Forte Marechal Luz (Ilha de São Francisco do Sul, SC) indicaram predomínio de indivíduos autóctones em ambos os níveis. Do conjunto de 32 indivíduos analisados, unicamente três não eram locais e provêm do interior, dois estavam enterrados no nível cerâmico do sambaqui, e um no nível pré-cerâmico. Estas análises também mostraram leves mudanças no padrão alimentar dos indivíduos enterrados no nível cerâmico do sítio, que os autores interpretam como resultado de maior deslocamento até o interior em busca de recursos.

No sítio misto praia da Tapera (Ilha de Santa Catarina), frequentemente associado a grupos Itararé (Silva et al. 1990; Fossari 2004; Schmitz et al. 1993), estudos sobre marcadores de estresse realizados por Scherer et al. (2006) indicam a prática de atividades vinculadas à captação de recursos marinhos (natação, remo, arremesso de redes, etc.). Esses mesmos indicadores foram amplamente identificados entre sambaquieiros de Santa Catarina e Rio de Janeiro (ver Mendonça de Souza 1995; Rodrigues-Carvalho 2003).

As evidências anteriores parecem indicar continuidade entre os indivíduos sepultados em sambaquis e sítios mistos e apontam a confirmar a primeira das hipóteses propostas por Wesolowski (2000). Assim como observado para os acampamentos litorâneos sem cerâmica, os sítios mistos teriam sido produzidos pela mesma população costeira e não necessariamente por migrantes ceramistas do interior que velozmente desenvolveram uma vida marítima, como já mencionado por Prous (1992).

\section{Domínio e miscigenação}

Existem duas diferenças maiores entre sambaquis e sítios mistos: a primeira refere-se ao padrão de residência pós-marital observado em cada tipo de sítio; a segunda, às marcas de violência detectadas nos indivíduos enterrados em sítios mistos.

Hubbe et al. (2009) identificaram que, em vários sítios mistos, entre os quais se incluem o da Tapera e Forte Marechal Luz, discutidos anteriormente, o padrão de residência é patrilocal, em oposição ao padrão matrilocal observado em sambaquis. O novo padrão de residência pode ser interpretado como resultado da chegada de novas populações. No entanto, entre os Jê meridionais do período histórico (Xokleng e Kaigang) menciona-se padrão de residência matrilocal (Urban 1978) e também bilateral (Santos, 1973), este último relacionado à reestruturação, produto das pressões exercidas pela sociedade nacional, como salienta Santos (1973). Portanto, Jê e sambaquieiros podem ter possuído o mesmo padrão de residência e a mudança observada em tempos tardios, uma resposta às pressões externas associadas com o aumento da influência Jê no litoral.

Em indivíduos sepultados em sítios mistos de Santa Catarina detecta-se um aumento nas marcas de violência (Lessa 2005; Lessa \& Scherer 2008). No entanto, outros estudos mostram que os indivíduos sepultados em sítios mistos apresentam as mesmas marcas osteológicas de atividade e a mesma dieta que os ocupantes dos sambaquis (Wesolowski, 2000; Scherer et al., 2006; Okumura, 2007; Bastos, 2009).

A mudança observada no padrão de residência e o aumento da violência seria mais significativa que a adoção da cerâmica, já que influi em vários aspectos da organização social e política. Isso significa que, apesar de predominar a continuidade genética e cultural entre ocupações cerâmicas no litoral e sambaquis, a influência interiorana há 1500-1000 anos foi mais complexa que a simples transmissão de uma inovação tecnológica como a cerâmica. Tal influência teria envolvido mudanças radicas na organização social, com intercâmbio de indivíduos, assim como aumento na violência inter e 
intragrupal (como mostrado por Lessa, 2005, e Lessa \& Scherer, 2008).

Aqui se propõe que o crescimento populacional e o poderio alcançado pelas populações do planalto, há 1500 anos, provocou a intensificação nas redes de interação entre planalto e litoral (ver Prous 1992). O novo domínio dos Jê sobre os sambaquieiros, que provocou uma intensa reestruturação e transformação do sistema sociocultural das comunidades litorâneas (ver Gaspar et al. 2007), pode inclusive ser uma resposta à pressão exercida pelos Guaranis sobre as populações interioranas (ver Noelli 1999, 2004). Assim, seriam estes indivíduos miscigenados no último milênio, sob o domínio da influência interiorana, os que encontraram os guaranis na costa, e não os descendentes de uma suposta leva migratória de grupos do planalto que se adaptaram rapidamente à vida litorânea há 1500-1000 anos.

\section{Conclusões}

O objetivo deste trabalho foi agrupar os resultados da produção científica sobre arqueologia dos sambaquis do litoral sudeste para sistematizar as informações e contribuir à formação de um panorama unificado sobre a pré-história do litoral. Como mencionado na introdução, este trabalho não pretende que o panorama unificado seja único ou exclusivo na explicação da pré-história do litoral sudeste. Este trabalho, de natureza essencialmente bibliográfica, expõe os mais recentes avanços das pesquisas na região com breves discussões sobre temas chave refrentes a povoamento da costa, relação dos sambaquis com outros sítios costeiros e abandono do projeto construtivo em conchas. As hipóteses levantadas refletem a visão da autora sobre os fenômenos discutidos e não necessariamente a conclusão final de discussões que ainda estão longe de ser esgotadas.

\section{Agradecimentos}

Agradeço o apoio financeiro da FAPESP (processo 08/51264-0) e do CNPq (processo 142532/2008-8). À Prof. Maria Dulce Gaspar (MN/UFRJ) pela leitura crítica da revisão bibliográfica aqui apresentada e ao Prof. Paulo C.F. Giannini (IGc/USP) pela revisão gramatical.

VILLAGRAN, X.S. What do we know about the sambaqui builders? Short review of the archaelogy of the southeastern coast of Brazil, from the first sambaquis to the arrival of Jê pottery. R. Museu Arq. Etn., São Paulo, n. 23, p. 139-154, 2013.

\begin{abstract}
From 1990 to the year of 2012 more than a hundred and fifty papers were written on the prehistory of the southeast coast of Brazil. Most of these works refer to the sambaqui (shell mound) sites, while another portion to the so called "coastal campsites", with or without ceramics, that characterize the recent prehistory of the coast. Within such a wide volume of work, how much do we actually know about the evolution of the human occupation of the coast and the characteristics of the groups that there inhabited before the massive migration of the Guarani groups? This paper aims to synthesize the most relevant information produced in the last twenty years on the prehistory of the southeast coast and to suggest new hypothesis on old questions related with the peopling of the coast and the "disappearance" of the sambaqui builders.
\end{abstract}

Key-words:: Coastal archaeology; Peopling of the coast; Coastal campsites; Taquara/Itararé pottery. 


\section{Referências bibliográficas}

ADAMS, J.

2002 Maritime archaeology. Encyclopedia of historical archaeology. 328-330.

AFONSO, M.C., DEBLASIS, P.

1994 Aspectos da formação de um grande sambaqui: alguns indicadores em Espinheiros II, Joinville. Revista do Museu de Arqueologia e Etnologia 4: 21-30.

ALVES, C.C.

2008 Análise zooarqueológica de um sambaqui fluvial: o caso do sítio Capelinha I. Universidade de São Paulo.

AMÂNCIO, S., DOMINGUEZ, J.M.L.

2003 Avaliação de áreas potenciais à presença de sambaquis na costa do estado de Sergipe utilizando como ponto de partida uma compreensão da evolução da zona costeira nos últimos 5600 anos AP. Canindé 3: 223-244.

AMENOMORI, S.N.

2005 Paisagem das ilhas, as ilhas da paisagem: a ocupação dos grupos pescadores-coletores pré-históricos no litoral norte do estado de São Paulo. Universidade de São Paulo.

BANDEIRA, D.

1992 Mudança na estratégia de subsistência. O sítio arqueológico Enseada I. Um estudo de caso. Universidade Federal de Santa Catarina.

BANDEIRA, D.

1999 Os sítios arqueológicos do litoral de Santa Catarina (Rio Lessa e Enseada I) e a problemática da tradiçao Taquara-Itararé vista através da alimentaçao. Revista do CEPA 23, 189-196.

BARBOSA, M.

2001 Espaço e organizaçao social entre o grupo social do sambaqui IBV4, Cabo Frio RJ.

BARBOSA, M. Universidade Federal do Rio de Janeiro.

2007 A ocupação pré-colonial da região dos lagos, RJ: sistema de assentamento e relações intersocietais entre grupos sambaquianos e grupos ceramistas tupinambá da

BARRETO, C. tradiçao Una. Universidade de São Paulo.

1988 A ocupaçao pré-colonial do vale do Ribeira de Iguape, SP: os sítios concheiros do médio curso. Universidade de São Paulo.

BARTOLOMUCCI, L.B.G.

2006 Variabilidade biológica de sambaquieiros através de morfologia dentária. Universidade de São Paulo.

BASTOS, M.

2009 Mobilidade humana na pré-história do litoral brasileiro: Análise de isótopos instáveis de estrôncio no sambaqui do Forte Marechal Luz. Fundaçao Oswaldo Cruz.

BASTOS, M., MENDONÇA DE SOUZA, S., SANTOS, R.V., LIMA, B., RODRIGUES-CARVALHO, C.

2011 Human mobility on the Brazilian coast : an analysis of strontium isotopes in archaeological human remains from Forte Marechal Luz sambaqui. Anais Da Academia Brasileira De Ciencias 83: 731-743.

BECK, A.

1971 Os sambaquis da região do litoral de Laguna - Santa Catarina. O homem pré-histórico na América. Instituto de Pré-história, Universidade de São Paulo, São Paulo.

BECK, A.

1972 A variação do conteúdo cultural dos sambaquis - litoral de Santa Catarina. Universidade de São Paulo.

BENDAZZOLI, C.

2007 O processo de formação dos sambaquis: uma leitura estratigráfica do sítio Jabuticabeira II, SC. Universidade de São Paulo.

BIANCHINI, G.F.

2008 Fogo e paisagem: evidências de práticas rituais e construção do ambiente a partir da análise antracológica de um sambaqui no litorral sul de Santa Catarina. Universidade Federal do Rio de Janeiro.

BIANCHINI, G.F., SCHEEL-YBERT, R., GASPAR, M.D.

2007 Estaca de Lauraceae em contexto funerário (sítio Jaboticabeira II, Santa Catarina, Brasil). Revista do Museu de Arqueologia e Etnologia 17: 223-229.

BIGARELLA, J.J.

1950 Contribuição ao estudo dos sambaquis no estado do Paraná. Arquivos de Biologia e Tecnologia V-VI: 231-345.

BROCHADO, J.P.

1984 An ecological model to the spread of pottery and agriculture into Eastern South America. University of Illinois.

BROCHIER, L.L.

2009 Controles geoarqueológicos e modelos morfoestratigráficos: implicaçoes para o estudo das ocupações pré-históricas na 
costa sul-sudeste do Brasil. Universidade de São Paulo.

BRYAN, A.L.

1993 The sambaqui at Forte Marechal Luz, state of Santa Catarina, Brazil. In: Bryan, A.L., Grhun, R. (Eds.), Brazilian Studies. Center for the study of the first Americans, Oregon

CALIPPO, F.R.

2004 Os sambaquis submersos de Cananeia: um estudo de caso de arqueologia subaquática. Universidade de São Paulo.

CALIPPO, F.R.

2010 Sociedade sambaquieira, comunidades marítimas. Universidade de São Paulo.

CANNON, A.

2000 Settlement and Sea-Levels on the Central Coast of British Columbia: Evidence from Shell Midden Cores. American Antiquity 65: 67-77.

CASTILHO, P.V. DE, SIMÕES-LOPES, P.C.

2008 Sea mammals in archaeological sites on the southern coast of Brazil. Revista do Museu de Arqueologia e Etnologia 18: 101- 113 .

CASTILHO, P. V.

2005 Mamíferos marinhos: um recurso de populaçoes humanas pré-coloniais do litoral catarinense. Universidade Federal do Paraná.

CRANCIO, F., KNEIP, L.M.

1994 Cultura material e subsistência das populaces pré-históricas de Saquarema, RJ. Documentos de Trabalho 2: 1-71.

DE MASI, M.A

1999 Mobility of prehistoric hunther-gatherers on southern Brazilian coast, Santa Catarina Island. University of Stanford.

DE MASI, M.A.

2001 Pescadores coletores da costa sul do Brasil. Pesquisas 57.

DEBLASIS, P., FISH, S.K., GASPAR, M.D., FISH, P.R.

1998 Some references for the discussion of complexity among the sambaqui moundbuilders from the southern shores of Brazil. Revista de Arqueologia Americana 15: 75-105.

DEBLASIS, P., KNEIP, A., SCHEEL-YBERT, R., GIANNINI, P.C.F., GASPAR, M.D.

2007 Sambaquis e paisagem: dinâmica natural e arqueologia regional no litoral sul do Brasil. Revista de Arqueología Sudamericana 3: 29-61.
DIAS, O.

1976 A evolução da cultura em Minas Gerais e no Rio de Janeiro. Anuário de Divulgação Científica 3: 110-130.

DIEGUES, C.A.

2004 A pesca construindo sociedades. NUPAUB USP, São Paulo.

DILLEHAY, T.D.

1999 Monte Verde: A Late Pleistocene Settlement in Chile, Volume 2: The Archaeological Context and Interpretation. Smithsonian Institution Press, Washingotn DC.

DILLEHAY, T.D.

2009 Probing deeper into first American studies. Proceedings of the National Academy of Sciences 106: 971-8.

DILLEHAY, T.D., BONAVIA, D., GOODBRED, S.L., PINO, M., VÁSQUEZ, V., THAM, T.R.

2012 A late Pleistocene human presence at Huaca Prieta, Peru, and early Pacific Coastal adaptations. Quaternary Research 77: 418-423,

DIXON, E.J.

2001 Human colonization of the Americas : timing, technology and process. Quaternary Science Reviews 20: 277-299.

DIXON, E.J.

2013 Late Pleistocene colonization of North America from Northeast Asia: New insights from large-scale paleogeographic reconstructions. Quaternary International 285: 57-67

ERLANDSON, J.M.

2001 The Archaeology of Aquatic Adaptations : Paradigms for a New Millennium. Journal of Archaeological Research 9: 287-350.

ERLANDSON, J.M., GRAHAM, M.H., BOURQUE, B.J., CORBETT, D., ESTES, J. A., STENECK, R.S.

2007 The Kelp Highway Hypothesis: Marine Ecology, the Coastal Migration Theory, and the Peopling of the Americas. The Journal of Island and Coastal Archaeology 2: 161-174.

ESCÓRCIO, E., GASPAR, M.D.

2010 Um olhar sobre gênero: estudo de caso sambaquieiros do RJ. Revista de Arqueologia SAB 23: 72-89.

FAGUNDES, N.J.R., KANITZ, R., ECKERT, R., VALLS, A.C.S., BOGO, M.R., SALZANO, F.M., SMITH, D.G., JR, W.A.S., ZAGO, M.A., RIBEIRO-DOS-SANTOS, A.K., SANTOS, S.E.B., PETZL-ERLER, M.L., BONATTO, S.L.

2008 Mitochondrial Population Genomics Supports a Single Pre-Clovis Origin with 
a Coastal Route for the Peopling of the Americas. American Journal of Human Genetics 82: 583-592

FIGUTI, L.

1992 Les sambaquis COSIPA (4200 à 1200 ans BP): étude de la subsistance chez les peuples préhistoriques de pêcheurs-ramasseurs de bivalves de la côte centrale de l'état de São Paulo, Brésil. Museum National d'Histoire Naturelle.

FIGUTI, L.

1993 O homem pré-histórico, o molusco e o sambaqui: considerações sobre a subsistência dos povos sambaquieiros. Revista do Museu de Arqueologia e Etnologia 3: 67-80.

FIGUTI, L., KLOKLER, D.

1996 Resultados preliminares dos vestígios zooarqueológicos do sambaqui Espinheiros II (Joinville, SC). Revista do Museu de Arqueologia e Etnologia 6: 169-188.

FILIPPINI, J.

2004 Biodistância entre sambaquieiros fluviais e costeiros: uma abordagem não métrica craniana entre três sítios fluviais do vale do Ribeira- SP (Moraes, Capelinha e Pavão XVI) e três costeiros do sul e sudeste do Brasil (Piaçaguera, Jabuticabeira II e Tenóri. Universidade de São Paulo.

FILIPPINI, J., EGGERS, S.

2005 Distância biológica entre sambaquieiros fluviais (Moraes - Vale do Ribeira - SP) e construtores de sítios litorâneos (Piaçaguera e Tenório - SP e Jabuticabeira II - SC). Revista do Museu de Arqueologia e Etnologia 15-16: 165-180.

FISH, S.K., BLASIS, P. DE, GASPAR, M.D., FISH, P.R.

2000 Eventos incrementais na construçao de sambaquis, litoral sul do estado de Santa Catarina. Revista do Museu de Arqueologia e Etnologia 10: 69-87.

FLADMARK, K.R.

1979 Routes: alternate migration corridors for early man in North America. American Antiquity 44: 55-69.

FOSSARI, T.D.

2004 A população pré-colonial Jê na paisagem da ilha de Santa Catarina.

GASPAR, M.D.

1991 Aspectos da organizaçao social de pescadores-coletores: região compreendida entre a Ilha Grande e o delta do Paraiba do Sul, Rio de Janeiro. Universidade de São Paulo.
GASPAR, M.D.

1998 Considerations of the sambaquis of the Brazilian coast. Antiquity 72: 592-615.

GASPAR, M.D.

2000 Sambaquis: arqueologia do Litoral. Jorge Zahar Editora, Rio de Janeiro.

GASPAR, M.D.

2004 Cultura: comunicaçao, arte, oralidade na pré-história do Brasil. Revista do Museu de Arqueologia e Etnologia 14: 153-168.

GASPAR, M.D., BARBOSA, D., BARBOSA, M.

1994 Análise do processo cognitivo de construção do Sambaqui Ilha da Boa Vista (RJ). Clio 1: 110-124.

GASPAR, M.D., BUARQUE, A., CORDEIRO, J., ESCÓRCIO, E.

2007 Tratamento dos Mortos entre os Sambaquieiros, Tupinambá e Goitacá que ocuparam a Região dos Lagos, Estado do Rio de Janeiro. Revista do Museu de Arqueologia e Etnologia 17: 169-189.

GASPAR, M.D., DEBLASIS, P.

1992 Construção de sambaquis: síntese das discussões do grupo de trabalho e colocaçao da proposta original. Anais da VI Reunião Científica da Sociedade de Arqueologia Brasileira. Rio de Janeiro, 811-820.

GASPAR, M.D., DEBLASIS, P., FISH, S.K., FISH, P.R.

2008 Sambaqui (Shell Mound) Societies of Coastal Brazil. In: Silverman, H., Isbell, W.H. (Eds.), Handbook of South American Archaeology. Springer, New York, 319-337.

GIANNINI, P.C.F., VILLAGRAN, X.S., FORNARI, M., RODRIGUES, D., MENEZES, P., TANAKA, A.P., ASSUNÇAO, D., DE BLASIS, P., AMARAL, P.

2010 Interações entre evolução sedimentar e ocupação humana pré-histórica na costa centro-sul de Santa Catarina, Brasil Interactions between sedimentary evolution and prehistoric human occupation in the south-central coast of Santa Catarina , Brazil. Bolteim do Museu Paraense Emílio Goeldi. Série Ciências Humanas 5: 105-128.

HUBBE, M., HARVATI, K., NEVES, W.

2011 Paleoamerican morphology in the context of European and East Asian late Pleistocene variation: implications for human dispersion into the New World. American journal of physical anthropology 144: 442-53

HUBBE, M., NEVES, W.A., CASTRO DE OLIVEIRA, E., STRAUSS, A.

2009 Postmarital residence practice in southern brazilian coastal groups: continuity and 
change. Latin American Antiquity 20: 267-278.

HURT, W.

1974 The interrelationships between the natural environment and four sambaqui, coast of Santa Catarina, Brazil. Indiana University Museum.

KARL, R.

2000 The Relative Chronology of Cultural Episodes at the Coastal Sambaqui, Jabuticabeira II, in Santa Catarina, Brazil. University of Arizona.

KLOKLER, D.M.

2001 Construindo ou deixando um sambaqui. Universidade de São Paulo.

\section{KLOKLER, D.M}

2008 Food for body and soul: mortuary ritual in shell mounds (Laguna - Brazil). University of Arizona.

KNEIP, A.

2004 O povo da lagoa: uso do SIG para modelamento e simulaçao na área arqueológica do Camacho. Universidade de São Paulo.

KNEIP, L.M.

1994 Cultura material e subsistência das populações pré-históricas de Saquarema, RJ. Documentos de Trabalho 2: 1-71.

LESSA, A.

2005 Reflexões preliminares sobre paleoepidemiologia da violência em grupos ceramistas litorâneos: I) sítio Praia da Tapera - SC. Revista do Museu de Arqueologia e Etnologia 15: 199-207.

LESSA, A., SCHERER, L.Z.

2008 O outro lado do paraíso: novos dados e reflexões sobre violência entre pescadores-coletores pré-coloniais. Revista do Museu de Arqueologia e Etnologia 18: 89-100.

LIMA, T.A.

1991 Dos mariscos aos peixes: um estudo zooarqueológico da mudança de subsistência ne pré-história do Rio de Janeiro. Universidade de São Paulo.

LIMA, T.A.

1999 Em busca dos frutos do mar: os pescadores-coletores do litoral centro-sul do Brasil. Revista da Universidade de São Paulo 44: 270-327.

LIMA, T.A., LOPEZ MAZZ, J.

1999 La emergencia de complejidad entre los cazadores recolectores da la costa atlántica meridional sudamericana. Revista de Arqueologia Americana 17, 18, 19: 129-175.
LIMA, T.A., MACARIO, K.D., ANJOS, R.M., GOMES, P.R.S., COIMBRA, M.M., ELMORE, D.

2002 The antiquity of the prehistoric settlement of the central-south brazilian coast. Radiocarbon 44: 733-738.

MANDRYK, C.A.S.C. A. ., JOSENHANS, H., FEDJE, D.W.D.W., MATHEWES, R.W.R.W.

2001 Late Quaternary paleoenvironments of Northwestern North America : implications for inland versus coastal migration routes. Quaternary Science Reviews 20: 301-314.

MENDONÇA DE SOUZA, A.

1991 História da Arqueologia Brasileira. Pesquisas 46.

MENDONÇA DE SOUZA, S.

1995 Estresse, doença e adaptabilidade. Estudo comparativo de dois grupos pré-históricos em perspectiva biocultural. Escola Nacional de Saúde Pública.

MIOTTI, L.

2003 Patagonia: a paradox for building images of the first Americans during the Pleistocene/Holocene Transition. Quaternary International 109-110: 147-173.

MIOTTI, L.L., SALEMME, M.

2004 Poblamiento, movilidad y territorios entre las sociedades cazadoras-recolectoras de Patagonia populations in Patagonia. Complutum 15: 177-206.

MUCKELROY, K.

1978 Maritime archaeology. Cambridge University Press, Cambridge.

NEVES, W.A.

1988 Paleogenética dos grupos pré-históricos do litoral sul do Brasil (Paraná e Santa Catarina). Pesquisas 43.

NEVES, W.A., HUBBE, M., OKUMURA, M.M., GONZÁlEZ-JOSÉ, R., FIGUTI, L., EGGERS, S., DE BLASIS, P.

2005 A new early Holocene human skeleton from Brazil: implications for the settlement of the New World. Journal of Human Evolutionman Evolution 48: 403-14.

NEVES, W.A., OKUMURA, M.M.M.

2005 Afinidades biológicas de grupos pré-históricos do vale do rio Ribeira de Iguape (SP): uma análise preliminar. Revista de Antropologia 48, 525-558.

NEVES, W.A., PROUS, A., GONZALEZ JOSE, R., KIPNIS, R., POWELL, J.

2003 Early Holocene human skeletal remains from Santana do Riacho, Brazil: implications for the settlement of the New 
World. Journal of Human Evolution 45: 19-42.

NEVES, W.A., WESOLOWSKI, V

2002 Economy, nutrition and disease in preshitoric coastal Brazil: a case study from the State of Santa Catarina. In: Steckel, R.H., Rose, J.C. (Eds.), The backbone of history. Cambridge University Press, Cambridge, 376-402.

NISHIDA, P.

2001 Estudo Zooarqueológico do Sítio Mar Virado Ubatuba - SP. Universidade de São Paulo.

NISHIDA, P.

2007 A coisa ficou preta: estudo do processo de formaçao da terra preta do sítio arqueológico Jabuticabeira II. Universidade de São Paulo.

NOELLI, F.S.

2000 Repensando os rótulos e a História dos Jê no Sul do Brasil a partir de uma interpretação interdisciplinar. In: Mota, L.T., Noelli, F.S., S., T. (Eds.), Uri e Wãxi - Estudos interdisciplinares dos Kaingang. Editora Universidade Estadual de Londrina, Londrina, 9-57.

OKUMURA, $\mathrm{M}$.

2007 Diversidade morfológica craniana, micro-evolução e ocupação pré-histórica da costa brasileira. Victoria. Universidade de São Paulo.

OKUMURA, M., EGGERS, S.

2005 Análise da exostose do meato auditivo externo como um marcador de atividade aquática em restos esqueletais humanos da costa e do interior do Brasil. Revista do Museu de Arqueologia e Etnologia 15, 16: 181-197.

OLIVEIRA, M.S.

2000 Os sambaquis da planície costeira de Joinville, litoral norte de Santa Catarina: geologia, paleogeografia e conservação in situ. Universidade Federal de Santa Catarina.

PERRETI, G.

2009 Sambaquis da barreira da Itapeva. Uma perspectiva geoarqueológica. Pontifícia Universidade Católica do Rio Grande do Sul.

PETRONILHO, C.C.

2005 Comprometimento articular como um marcador de atividade em um grande sambaqui cemitério. Universidade de São Paulo.
PLENS, C.R.

2007 Sítio Moraes, uma biografia não autorizada: análise do processo de formaçao de um sambaqui fluvial. Universidade de São Paulo.

PROUS, A.

1992 Arqueologia Brasileira. Universidade de Brasilia, Brasilia.

RODRIGUES-CARVALHO, C.

2003 Marcadores de estresse ocupacional em populações sambaquieiras no litoral fluminense. Escola Nacional de Saúde Pública.

ROHR, P.J.A.

1984 Sítios arqueológicos de Santa Catarina. Anais do Museu de Antropologia 17: 77-168.

ROTHHAMMER, F., DILLEHAY, T.D.

2009 The late Pleistocene colonization of South America: an interdisciplinary perspective. Annals of Human Genetics 73: 540-9,

SANDWEISS, D.H.

2003 Terminal Pleistocene through Mid-Holocene archaeological sites as paleoclimatic archives for the Peruvian coast. Palaeogeography, Palaeoclimatology, Palaeoecology 194: 23-40.

SANTOS, S.C.

1973 Índios e Brancos no Sul do Brasil - a dramática experiência dos Xokleng. Edeme, Florianópolis.

SCHERER, L.Z., RODRIGUES-CARVALHO, C., SCHMITZ, P.I.

2006 Marcadores de estresse musculoesquelético em populações pescadoras, caçadoras e coletadas do litoral central de Santa Catarina. Pesquisas 63: 55-80.

SCHMITZ, P.I.

1999 Cerâmica Taquara e Itararé em concheiros. Revista do CEPA 23: 177-179.

SCHMITZ, P.I., BITTENCOURT, A.L.V.

1996 O sítio arqueológico do Pântano do Sul, S.C. Pesquisas 53: 77-124.

SCHMITZ, P.I., DE MASI, M.A., VERARDI, I., LAVINA, R., JACOBUS, A.L.

1992 Escavações arqueológicas do Pe. João Alfredo Rohr, S.J. O sítio arqueológico da Armação do Sul. Pesquisas 48.

SCHMITZ, P.I., DE MASI, M.A., VERARDI, I., LAVINA, R., JACOBUS, A.L.

1993 Escavações arqueológicas do Pe. João Alfredo Rohr, S.J. O sítio arqueológico Praia das Laranjeiras II, uma aldeia de tradição ceramista Itararé. Pesquisas 49.

SCHMITZ, P.I.

1996 Visão de conjunto dos sítios da Tapera, Armação do Sul, Laranjeiras I e II, Pân- 
tano do Sul e Cabeçudas. Pesquisas: 53, 183-190.

SILVA, S.B., SCHMITZ, P.I., ROGGE, J., DE MASI, M.A., JACOBUS, A.L., BATISTA DA SILVA, S.

1990 Escavações arqueológicas do Pe. João Rohr - O sítio arqueológico da praia da Tapera: um assentamento Itararé e Tupi-guarani. Pesquisas 45.

TENÓRIO, M.C.

2003 Dinâmica de ocupação pré-histórica no litoral brasileiro. Universidade Católica do Rio Grande do Sul.

TENÓRIO, M.C.

2004 Identidade cultural e origem dos sambaquis. Revista do Museu de Arqueologia e Etnologia 14: 169-178.

TENÓRIO, M.C., PINTO, D., AFONSO, M.C.

2008 Dinâmica de ocupação, contatos e trocas no litoral do Rio de Janeiro no período de 4000 a 2000 anos antes do presente. Arquivos do Museu Nacional 66: 311-321.

TIBURTIUS, G.

1996 A Coleção de Guilherme Tiburtius. Arquivos de Guilherme Tiburtius I. Fundação Cultural de Joinville, Museu Arqueológico de Sambaqui de Joinville, Joinville, $13-28$.

URBAN, G.

1992 A História da Cultura Brasileira Segundo as Linguas Nativas. História dos Índios no Brasil. Companhia das Letras, São Paulo, 87-102.

VILLAGRAN, X.S.

2008 Análise de arqueofácies na camada preta do sambaqui Jabuticabeira II. Universidade de São Paulo.
VILLAGRAN, X.S.

2010 Geoarqueologia de um sambaqui monumental: Estratigrafias que falam. Annablume Editora, São Paulo.

VILLAGRAN, X.S., DEBLASIS, P., GIANNINI, P.C.F.

2009 Primeros estudios micromorfológicos en sambaquís brasileños (sitio Jabuticabeira II, Estado de Santa Catarina). Intersecciones en Antropología 10: 359-364.

VILLAGRAN, X.S., GIANNINI, P.C.F., DEBLASIS, P.

2009b. Archaeofacies analysis: Using depositional attributes to identify anthropic processes of deposition in a monumental shell mound of Santa Catarina State, southern Brazil. Geoarchaeology: 24, 311-335.

VILLAGRAN, X.S., KLOKLER, D., NISHIDA, P., GASPAR, M.D., DE BLASIS, P.

2010 Lecturas estratigraficas: arquitetura funeraria y depositación de residuos en el sambaquí Jabuticabeira II. Latin American Antiquity: 21, 195-216.

WESOLOWSKI, V.

2000 A prática da horticultura entre os construtores de sambaquis e acampamentos litorâneos da região da Baía de São Francisco, Santa Catarina: uma abordagem bioantropológica. Universidade de São Paulo.

WESOLOWSKI, V., MENDONÇA DE SOUZA, S., REINHARD, K., CECCANTINI, G.

2007 Grânulos de amido e fitólitos em cálculos dentários humanos: contribuição ao estudo do modo de vida e subsistência de grupos sambaquianos do litoral sul do Brasil. Revista do Museu de Arqueologia e Etnologia: 17, 191-210. 